\title{
Ischemia induces cell proliferation and neurogenesis in the gerbil hippocampus in response to neuronal death
}

\author{
Pablo Salazar-Colocho*, José L. Lanciego, Joaquin Del Rio, Diana Frechilla \\ Division of Neuroscience, CIMA, University of Navarra, Av. Pío XII 55, 31008-Pamplona, Spain \\ Received 20 September 2007; accepted 11 January 2008 \\ Available online 29 January 2008
}

\begin{abstract}
We studied hippocampal cellular proliferation and neurogenesis processes in a model of transient global cerebral ischemia in gerbils by labelling dividing cells with 5'-Bromo-2'-deoxyuridine (BrdU). Surrounding the region of selective neuronal death (CA1 pyramidal layer of the hippocampus), an important increase in reactive astrocytes and BrdU-labelled cells was detected 5 days after ischemia. A similar result was found in the dentate gyrus (DG) 12 days after ischemia. The differentiation of the BrdU+ cells was investigated 28 days after BrdU administration by analyzing the morphology, anatomic localization and cell phenotype by triple fluorescent labelling (BrdU, adult neural marker NeuN and DNA marker TOPRO-3) using confocal laser-scanning microscopy. This analysis showed increased neurogenesis in the DG in case of ischemia and triple positive labelling in some newborn cells in CA1. Seven brain hemispheres from gerbils subjected to ischemia did not develop CA1 neuronal death; hippocampus from these hemispheres did not show any of the above mentioned findings. Our results indicate that ischemia triggers proliferation in CA1 and neurogenesis in the DG in response to CA1 pyramidal neuronal death, independently of the reduced cerebral blood flow or the cell migration from subventricular zone (SVZ).
\end{abstract}

(C) 2008 Elsevier Ireland Ltd and the Japan Neuroscience Society. All rights reserved.

Keywords: BrdU; Confocal laser scanning microscopy; Global cerebral ischemia; Hippocampal CA1 region; Neurogenesis

\section{Introduction}

Stroke is the third most common cause of death in developed countries, exceeded only by coronary heart disease and cancer; also, represents the first cause of permanent disability above the age of 65 years. Despite that a restorative treatment has not been reached yet.

The adult central nervous system contains stem cells that are capable of generating new neurons, astrocytes and oligodendrocytes (Emsley et al., 2005). It has been suggested that increased neurogenesis originating from stem cells located in subventricular zone (SVZ) and subgranular zone (SGZ) of the hippocampal dentate gyrus (DG) of adult brain in a variety of mammals, including humans, may promote morphological and functional recovery after cerebral ischemia, traumatic and primary degenerative brain diseases (Gould et al., 1999; Schmidt and Reymann, 2002).

\footnotetext{
* Corresponding author. Tel.: +34 948 194700; fax: +34 948194715 .

E-mail addresses: pscolocho@alumni.unav.es, pabloesalazar@gmail.com (P. Salazar-Colocho).
}

It is known that transient global cerebral ischemia leads to delayed cell death of CA1 pyramidal neurons of the hippocampus in adult gerbils (Kirino, 1982; Schmidt-Kastner and Freund, 1991; Hara et al., 1993; Ferrer et al., 1997; Horiguchi et al., 2002), and that brain ischemia increases neurogenesis in the DG of the hippocampus of rodents including gerbils (Takagi et al., 1999; Sharp et al., 2002; Kokaia and Lindvall, 2003).

The origin of neural stem cells that give rise to new neurons in the adult CNS has been a subject of debate. Although neural progenitor proliferation and neurogenesis occur in SVZ and SGZ, several studies have demonstrated the production of new neurons in many other regions of the adult mammalian brain including CA1 area of gerbils after global cerebral ischemia (Schmidt and Reymann, 2002).

This and other issues like the mechanisms that trigger and regulate the proliferation and differentiation of neuronal progenitors are important to understand adult neurogenesis. Given the potential clinical applications and the limited understanding of this process, we decided to investigate the proliferation of progenitor cells and neurogenesis in the dentate 
gyrus and CA1 area of hippocampus at different time points after transient global cerebral ischemia by labelling dividing cells with the synthetic thymidine analogue BrdU, which is incorporated into the DNA of dividing cells in the S phase of the cell cycle and can be detected immunohistochemically (Gratzner, 1982; Kuhn et al., 1996). The expression of the glial marker Glial Fibrillary Acidic Protein (GFAP) was also studied. To determine the survival and fate of proliferating cells in the granule cell layer (GCL) of the DG and CA1 newborn cells were examined 4 weeks after BrdU administration using confocal microscopy.

Transient global cerebral ischemia was induced by occlusion of both common carotid arteries (CCA) for $5 \mathrm{~min}$. In the gerbil brain, the lack of posterior communicating arteries between the carotid arteries and the vertebral arteries constitutes an incomplete Circle of Willis. Due to this unique anatomical structure, the gerbil has been widely used as an animal model to produce transient global cerebral ischemia following occlusion of both CCA (Ginsberg and Busto, 1989). However, because of the presence of substantial genetic heterogeneity in communicating arteries in gerbils, some animals have partial communicating vessels between the basilar and carotid circulations on one side (Wang et al., 2002). According to this and our results, hippocampal CA1 pyramidal neuronal death triggers proliferation in CA1 and neurogenesis in the DG, independently of the reduced blood flow.

\section{Materials and methods}

\subsection{Animal experimental design and BrdU injections}

Procedures involving animals were in accordance with the guidelines established by the European Communities Council Directive of 24 November 1986 (86/609/EEC).

For proliferation studies, adult male mongolian gerbils (Meriones unguiculatus; 11-12 weeks of age; Harlan, Barcelona, Spain) weighing 60-65 g were divided into two groups: animals subjected to $5 \mathrm{~min}$ of global cerebral ischemia $(n=21)$ and sham operated animals $(n=15)$. Each group of gerbils was sacrificed (perfused transcardially) at three different time points after ischemia-reperfusion: 5,12 and 21 days ( $n=5-7$ animals per group and time point). Twenty-four hours before sacrifice, all animals received a single intraperitoneal injection of BrdU ( $50 \mathrm{mg} / \mathrm{kg}$ dissolved in $0.9 \% \mathrm{NaCl}$; Sigma-Aldrich, Steinheim, Germany).

For neurogenesis studies and according to our findings in the proliferation study, two groups of gerbils were divided as follows. $A$ : sham control $(n=5)$ and ischemic animals $(n=7)$ received daily two intraperitoneal injections of BrdU $(50 \mathrm{mg} / \mathrm{kg}$ ) during days $4,5,6$ and 7 post-ischemia and were allowed to survive for 28 days after the first BrdU injection ( 32 days after surgery). $B$ : sham control $(n=5)$ and ischemic animals $(n=7)$ received daily two intraperitoneal injections of BrdU (same dosage) during days 10, 11, 12 and 13 post-ischemia and were allowed to survive for 28 days after the first BrdU injection (38 days after surgery). Animals were then anesthetized and perfused.

\subsection{Transient global cerebral ischemia}

Gerbils were anesthetized with Equithesin (chloral hydrate 4.26\%, sodium pentobarbital $0.96 \%$ and magnesium sulfate $2.12 \%$, in aqueous solution with $9.5 \%$ ethanol) $3 \mathrm{ml} / \mathrm{kg}$, i.p. (Gaese and Ostwald, 1995, 2001). After a medial neck incision, both CCA were exposed and occluded with aneurysm clips for $5 \mathrm{~min}$. The clips were then removed to restore cerebral blood flow. The rectal temperature was controlled with a heating blanket to prevent hypothermia. Sham operated animals were treated identically, except that CCA were not occluded after the neck incision and carotid exposure.

\subsection{Fixation and tissue processing}

After anaesthesia animals were perfused transcardially with $100 \mathrm{ml}$ of saline and $200 \mathrm{ml}$ of $4 \%$ paraformaldehyde in phosphate-buffered saline (PBS). After perfusion, all brains were removed, post-fixed in the same fixative solution for $1 \mathrm{~h}$ at room temperature and cryoprotected in $30 \%$ sucrose solution in phosphate buffer (PB) overnight at $4{ }^{\circ} \mathrm{C}$. Freezing microtome sections (30$\mu \mathrm{m}$-thick) were cut coronally through the entire hippocampus and SVZ, in series of 5 adjacent sections, collected free-floating and stored in $30 \%$ ethylene glycol, $30 \%$ glycerol, and $0.1 \mathrm{M} \mathrm{PB}$ at $-20{ }^{\circ} \mathrm{C}$ until processed for immunostaining. Some sections were processed for staining with thionin (Nissl staining) and diaminobenzidine (DAB) NeuN immunohistochemistry for histological assessment of neuronal death.

\subsection{Immunohistochemistry}

\subsubsection{Immunofluorescence}

Four free floating tissue sections comprising the hippocampal formation of five animals per group were processed for immunohistochemistry (20 different sections per group). For BrdU staining, sections were pre-treated for DNA denaturalization in $2 \mathrm{~N} \mathrm{HCl}$ for $30 \mathrm{~min}$ at $37^{\circ} \mathrm{C}$ and then rinsed in borate buffer $0.1 \mathrm{M}(\mathrm{pH} 8.5)$ for $10 \mathrm{~min}$. Brain sections were washed $(3 \times 10 \mathrm{~min})$ with PBS $0.1 \mathrm{M}(\mathrm{pH} 7.4)$ and incubated in blocking solution (PBS containing $0.3 \%$ Triton $\mathrm{X}-100,0.1 \% \mathrm{BSA}$ and $2 \%$ normal goat serum) for $2 \mathrm{~h}$ at room temperature. Primary and secondary antibodies were diluted in the blocking solution. Sections were incubated with the primary antibodies for $24 \mathrm{~h}$ at $4{ }^{\circ} \mathrm{C}$, washed with PBS and incubated with the secondary antibodies for $2 \mathrm{~h}$ at room temperature, protected from light. The primary antibodies and the concentration used were: monoclonal rat anti BrdU (1:300, Accurate Chemical, Westbury, NY, USA); monoclonal mouse anti GFAP (1:500, Sigma, St. Louis, MO, USA); monoclonal mouse anti NeuN (1:500, Chemicon, Temecula, CA, USA). Secondary antibodies used were Alexa fluor 488 donkey anti-rat and Alexa fluor 546 goat anti-mouse highly cross-adsorbed (both 1:200, InvitrogenMolecular Probes, Eugene, Oregon, USA). For double labelling, sections were incubated at the same time with both primary antibodies and then simultaneously with the both respective secondary antibodies. Sections were mounted on SuperFrost plus microscope slides (Menzel GmbH \& Co KG, Braunschweig, Germany), air dried for $24 \mathrm{~h}$, rinsed in toluene $(2 \times 5 \mathrm{~min})$, and cover-slipped with DPX mounting medium. For better visualization of nuclei, sections were rinsed 2 min in the DNA marker TOPRO-3 (Suzuki et al., 1997; Martin et al., 2005), working concentration $4 \mu \mathrm{M}$ in PBS (Invitrogen-Molecular Probes, Eugene, Oregon, USA), and then washed 2 min in PBS after mounting.

To ensure comparable immunostaining, sections were processed together at the same time under the same conditions. For the assessment of non-specific primary immunostaining, sections from each experimental group were incubated without the primary antibodies; in this case no immunostaining was observed. We also evaluated non-specific secondary immunostaining incubating sections with primary and its non-respective secondary antibody; again, no immunostaining was observed. Fluorescence signals were detected with Nikon eclipse 800 microscope (Nikon, Japan) and confocal microscope LSM 510 Meta (Carl Zeiss, Germany).

\subsubsection{DAB immunohistochemistry}

For DAB NeuN immunostaining, free floating sections (2 per animal) were rinsed in several changes of PBS $0.1 \mathrm{M}(\mathrm{pH} 7.4)$, treated with $1 \%$ hydrogen peroxide in PBS during $15 \mathrm{~min}$, preincubated for $2 \mathrm{~h}$ in blocking solution (PBS $0.1 \mathrm{M}$, pH 7.4, containing $0.3 \%$ Triton $\mathrm{X}-100,2 \%$ normal goat serum and $0.1 \%$ BSA) and then incubated overnight at $4{ }^{\circ} \mathrm{C}$ with the primary antibody, monoclonal mouse anti NeuN (1:200, Chemicon, Temecula, CA, USA) in blocking solution. The sections were then washed in PBS three times and incubated with the secondary antibody, biotinylated goat-anti-mouse (1:200; Vector Lab., CA, USA), for $2 \mathrm{~h}$ at room temperature. Sections were again rinsed in PBS three times and the subsequent visualization procedure was performed by the avidinbiotin complex (ABC) method (Vectastain ABC Kit; Vector Lab., CA, USA) with diaminobenzidine (DAB) as chromogen (peroxidase substrate kit, Vector Lab.). Sections were mounted on super frost plus slides, air dried for $24 \mathrm{~h}$, dehydrated, cleared with xylene and cover-slipped with DPX mounting med- 
ium. Microphotographs were captured with Nikon eclipse 800 microscope (Nikon, Japan).

\subsection{Quantification}

BrdU-positive cells from single labelled sections were counted along all SGZ of the dentate gyrus from five animals per group, four sections per animal (20 different sections per group) using $20 \times$ objective and the image analyzer software "analySIS" (Soft Imaging System GmbH, 2003, Germany); the values were averaged and expressed as mean number of BrdU+ cells in SGZ of the DG.

Fluorescent double and triple labelling was observed in slices using confocal microscopy under $40 \times$ and $63 \times$ objectives. All double and triple labelling colocalization was confirmed by $3 \mathrm{D}$ analysis rotating the images along each axis and evaluating the sections in Z-series ( $\sim 0.5 \mu \mathrm{m}$ steps) using LSM 510
Meta software (Carl Zeiss, Germany). We analyzed 287 BrdU+ cells from BrdU-GFAP labelled sections in CA1 subfield 5 days after ischemia (5 animals, 3 sections per animal). For asses neurogenesis we perform a semi-quantification of the triple positive cells (BrdU-NeuN-TOPRO-3) in the granule cell layer (GCL) of the DG; triple positive cells were counting in a sampling area of $20037.19 \mu \mathrm{m}^{2}$ of GCL using LSM 510 Meta software (Fig. 7A) from randomised sections of four groups of study: sham 32 and 38 days after surgery; ischemia 32 and 38 days after CCA occlusion.

\subsection{Statistical analysis}

Results, reported as means \pm S.E.M., were analyzed using one-way ANOVA followed by post hoc comparisons with the Scheffé test. The level of statistical significance was set at $p<0.05$.
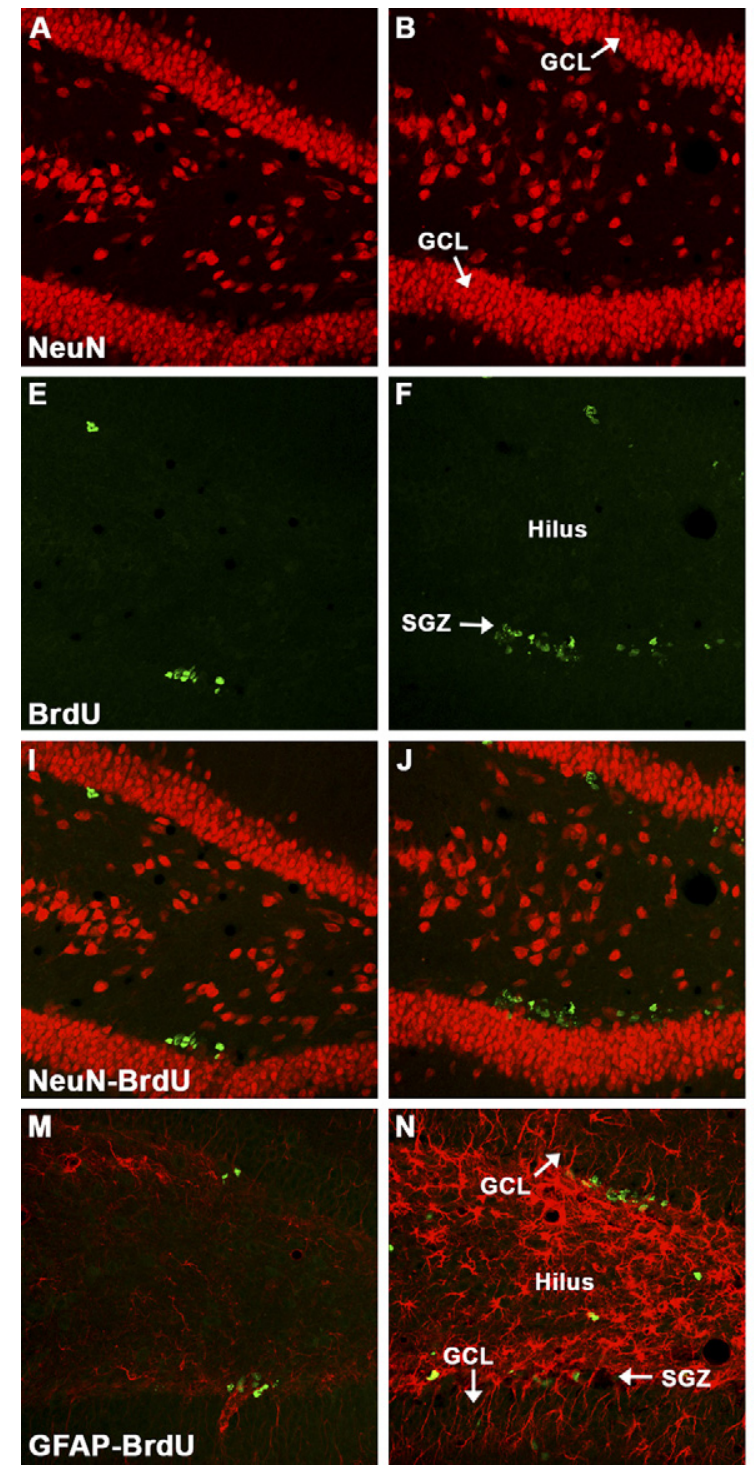
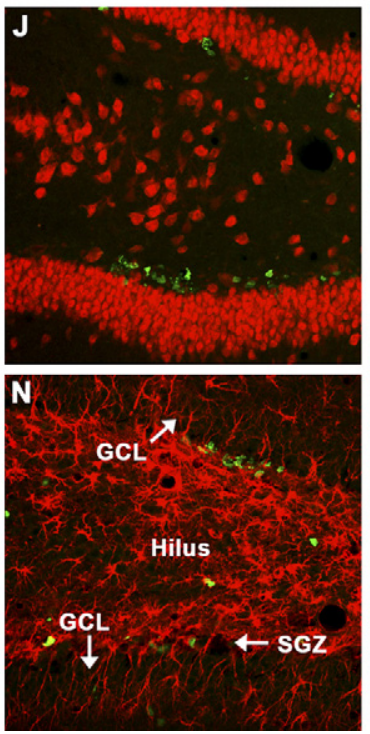
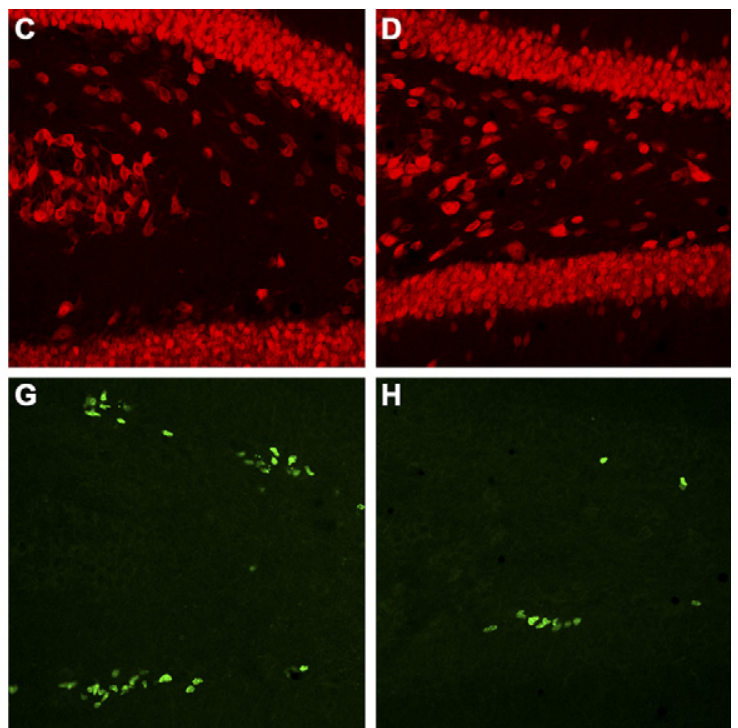

H
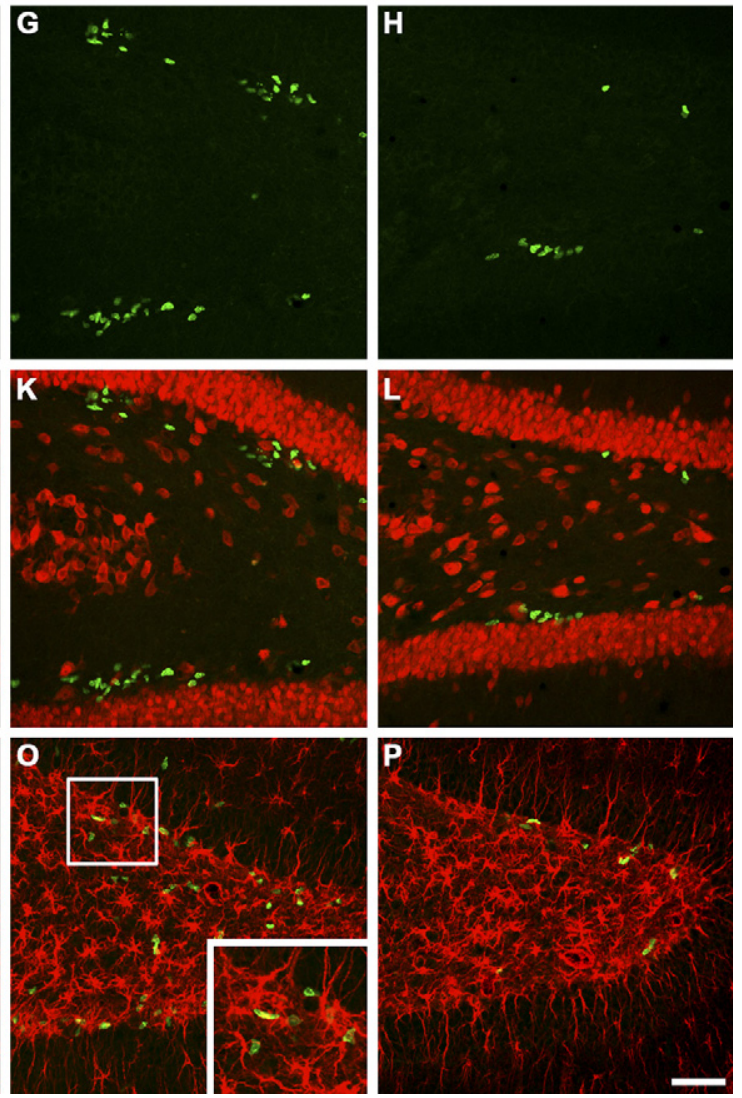

Fig. 1. Proliferation in the dentate gyrus (DG). (A-L) Representative confocal images of the DG of hippocampus showing immunofluorescent double labelling for NeuN (red), BrdU (green) and merged (NeuN-BrdU). (M-P) Representative confocal images of the immunofluorescent double labelling for GFAP (red)-BrdU (green) showing similar areas of the DG. (A, E, I, M) Sham-control animals; (B, F, J, N) 5 days after ischemia; (C, G, K, O) 12 days after ischemia; (D, H, L, P) 21 days after ischemia. NeuN stain shows adult neurons in GCLs and hilus of the DG and BrdU labelling shows proliferative cells in SGZ of the DG. Note the increased cell proliferation 5 and 12 days after ischemia in SGZ ( $F$ and G, respectively) compared to sham (E) and that this reaction is maximal at 12 days $(\mathrm{G})$. Also note that the BrdU+ cells in SGZ do not correspond to astrocytes (M-P) because they do not colocalize with GFAP and do not have astrocytic morphology (the absence of colocalization GFAP-BrdU in the SGZ BrdU+ cells was confirmed by scanning through three planes). The right insert in O is a higher-magnification view of the box in the same panel showing that there is no GFAP-BrdU colocalization. There is a marked increase of GFAP immunoreactivity in the hilus of the DG at all time point after ischemia (N-P) compared to sham (M). GCL: granular cell layer, SGZ: subgranular zone. Scale bar $=50 \mu \mathrm{m}$ (For interpretation of the references to color in this figure legend, the reader is referred to the web version of the article.). 


\section{Results}

\subsection{Proliferation studies}

Proliferation was defined as the number of newborn cells measured $24 \mathrm{~h}$ after a single injection of BrdU. Ischemic animals showed an increase in the number of proliferating cells in SGZ of the DG at 5 and 12 days after ischemia; these BrdU+ cells were not astrocytes because they did not express GFAP (Fig. 1). The number of BrdU+ cells in SGZ of the sham animals 5 days after surgery was $18.7 \pm 2.5$ (mean \pm S.E.M.), this number increased to $34.6 \pm 2.2$ in the ischemic animals 5 days after ischemia, $p<0.05$ (one-way ANOVA followed by Sheffé test). The number of BrdU+ cells in SGZ of the sham animals 12 days after surgery was $20.1 \pm 2.2$, which increased to $47.5 \pm 2.5$ in the ischemic animals 12 days after ischemia, $p<0.05$ (one-way ANOVA followed by Sheffé test). The increase in the number of BrdU+ cells in case of ischemia was maximal at 12 days after ischemia $47.5 \pm 2.5, p<0.05$ vs. the 5 day time point, $34.6 \pm 2.2$ (one-way ANOVA followed by
Sheffé test). Then, ischemia increased, significantly, the cell proliferation in SGZ of the DG at 5 and 12 days after ischemia and a maximal increase was observed 12 days after ischemia (Fig. 2A).

In previous studies we corroborated clearly the delayed neuronal death in CA1 pyramidal cell layer after 5 min of bilateral CCA occlusion in our model (Fig. 2B). A remarkable cellular proliferation was found in CA1 5 days after ischemia compared to sham controls. This cell proliferation was not observed either 12 or 21 days after ischemia in this hippocampal subfield. To ensure that our finding in CA1 corresponded to gerbils that really developed neuronal death in this area we performed this part of the study on sections with double labelling for BrdU and NeuN. Only sections where neuronal death was present (demonstrated by loss of NeuN labelling in CA1) showed the increase in cell proliferation 5 days after ischemia (Fig. 3).

We also examined the SVZ 5, 12 and 21 days after ischemia (data not shown), looking for an eventual migration from this well-known proliferative zone to the hippocampus. We did not

(A)

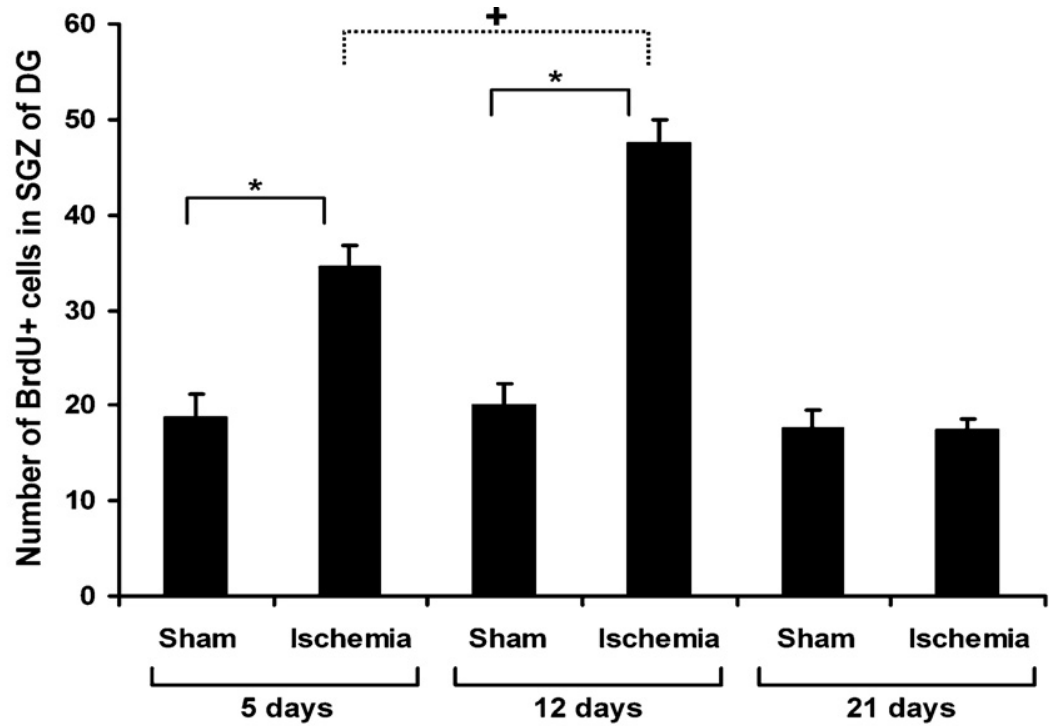

(B)
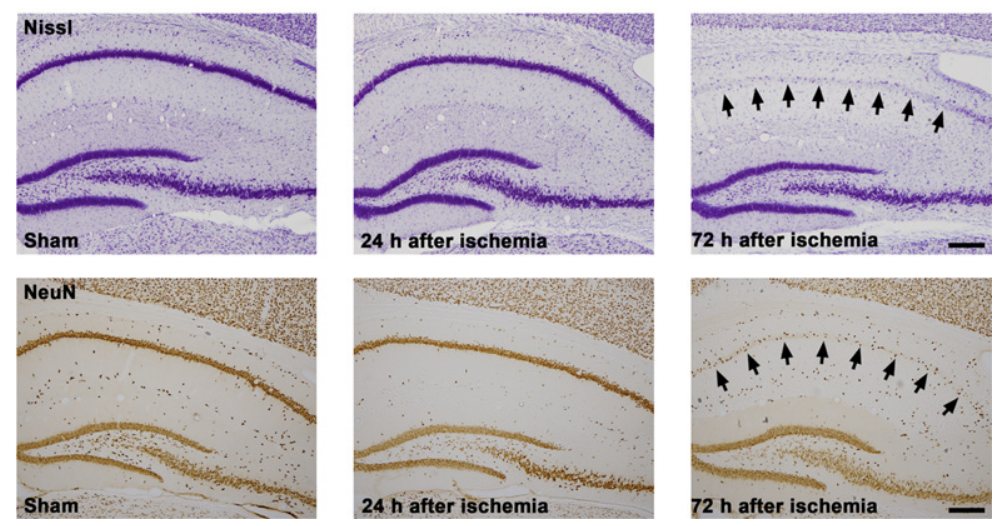

Fig. 2. (A) Counting of BrdU+ cells in SGZ. The histogram shows the number of BrdU labelled cells in SGZ of the dentate gyrus at different time points in sham controls and ischemic animals. The values indicate absolute counts (mean \pm S.E.M.) per dentate gyrus. ${ }^{*} p<0.05$ vs. the corresponding sham controls, ${ }^{+} p<0.05$ vs. the 5 day time point (one-way ANOVA followed by Sheffé test). (B) Delayed CA1 neuronal death after ischemia. Nissl-stained sections and NeuN immunohistochemistry using diaminobenzidine. Note the extensive and clear delayed neuronal loss of CA1 pyramidal neurons $72 \mathrm{~h}$ after ischemia (indicated by arrows) compared with sham or ischemic animals $24 \mathrm{~h}$ after the ischemic insult. Scale bars $=250 \mu \mathrm{m}$. 

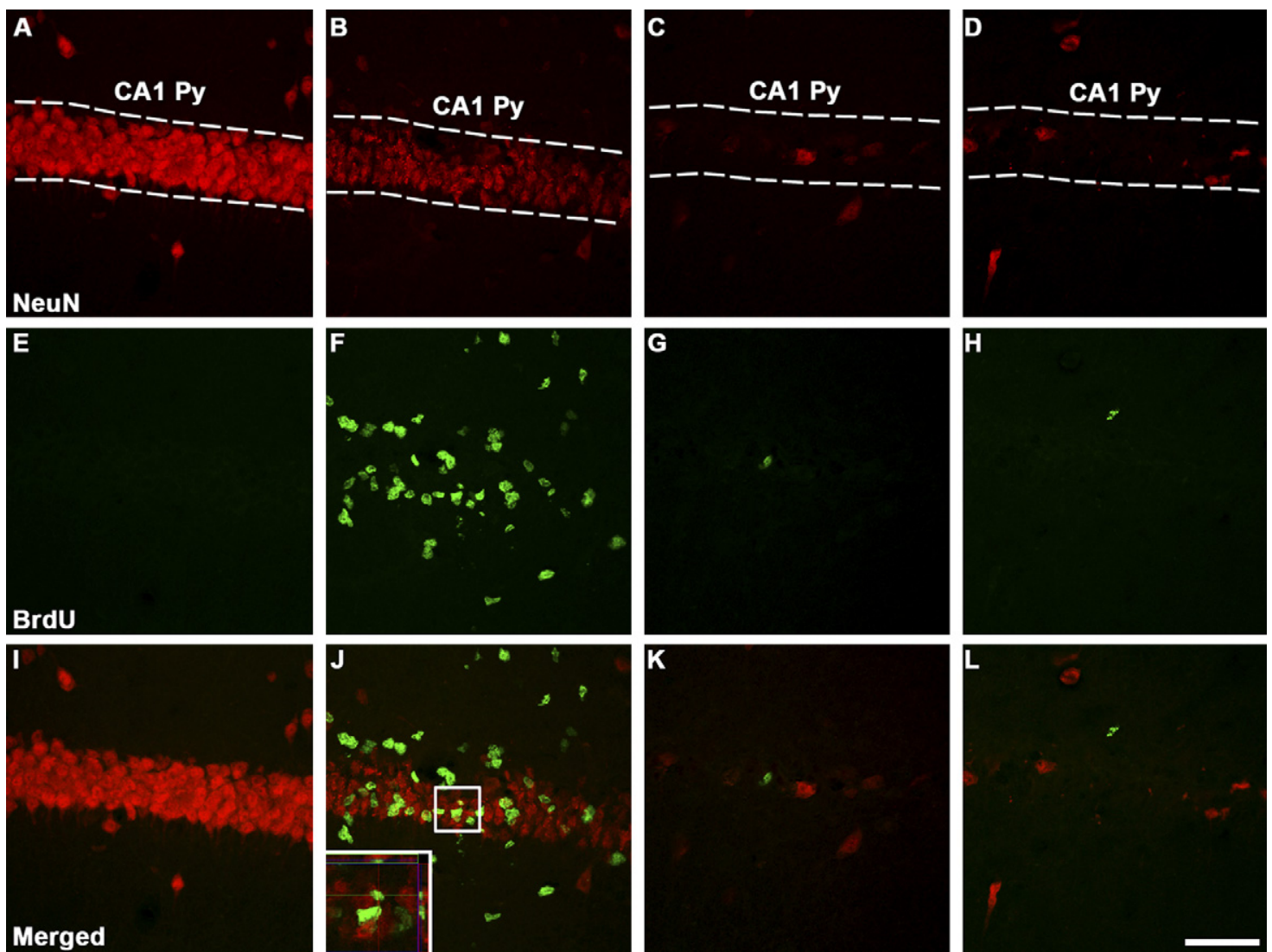

Fig. 3. Proliferation in CA1. Representative confocal images from CA1 area of the hippocampus, showing immunofluorescent staining for NeuN (red), BrdU (green) and merged. NeuN stain shows adult neurons in CA1 pyramidal cell layer (CA1 Py, indicated by dashed lines) and BrdU labelling shows proliferative cells. (A, E, I) Sham-control animals; (B, F, J) 5 days after ischemia; (C, G, K) 12 days after ischemia; (D, H, L) 21 days after ischemia. Note the loss of NeuN staining in ischemic animals (B-D) compared to sham (A) and the marked increase of cell proliferation in CA1 5 days after ischemia (F). Also note that there is no colocalization of NeuNBrdU in any cell $24 \mathrm{~h}$ after BrdU injection $(\mathrm{J}-\mathrm{L})$. The left insert in $(\mathrm{J})$ is a higher magnification view and $3 \mathrm{D}$ analysis of the central smallest BrdU+ cell in the box, demonstrating that this cell is in a different $\mathrm{Z}$ plane of the NeuN stained cells. Scale bar $=50 \mu \mathrm{m}$ (For interpretation of the references to color in this figure legend, the reader is referred to the web version of the article.)

find however any important difference in proliferating cells between sham-controls and ischemic animals at any time point; only a few ectopic cells were found 5 and 12 days after ischemia.

There was also a marked astrocytic reaction in CA1 in all sections from gerbils subjected to cerebral ischemia and showing delayed pyramidal neuronal death, this reaction was independently of time after ischemia (Fig. 4B and D). Given the marked cell proliferation found in CA1 5 days after ischemia and the increase in GFAP immunoreactivity at all time points, double immunohistochemistry for GFAP and BrdU was also performed in sections from animals sacrificed 5 days after ischemia in order to identify eventual astrocytic features of newborn cells at this time. We analyzed $287 \mathrm{BrdU}+$ cells from the group "ischemia": $32 \%$ of $\mathrm{BrdU}(+)$ cells showed colocalization with GFAP, i.e. approximately one third of the newborn cells found in CA1 5 days after ischemia were astrocytes (Fig. 4E and F).

\subsection{Neurogenesis studies}

The presence of newly born neurons was demonstrated analyzing the morphology, anatomic localization and cell phenotype by triple fluorescent labelling (NeuN-BrdU-TOPRO-3) of the newborn cells 28 days after the first BrdU injection, a long enough time for neuronal differentiation. We found triple NeuNBrdU-TOPRO-3 positive cells with typical normal neuronal morphology and anatomic localization of the granule neurons of GCL of the DG, indicating neurogenesis in this cerebral area (Fig. 5A-H).

In basal conditions, there was no neurogenesis in CA1 (Fig. 5I-L). However, triple colocalization of NeuN-BrdUTOPRO-3 was also observed in CA1 pyramidal layer 32 days after the transient ischemic insult (Fig. 5M-P). We found one or two of these cells along ischemic CA1 pyramidal layer (Fig. 6A-F). The colocalization of neurogenesis markers in CA1 was found in ischemic animals that received injections of BrdU during days 4, 5, 6 and 7 post-ischemia and was not observed in ischemic animals that received injections of BrdU during days 10,11, 12 and 13 post-ischemia.

Interestingly, seven brains of different ischemic animals (from all ischemic groups) developed CA1 pyramidal neuronal death only in one hemisphere; i.e. seven gerbils subjected to 5 min of bilateral CCA occlusion did not show CA1 pyramidal neuronal death in one brain hemisphere. In the hippocampus from hemispheres without neuronal death, neither proliferation nor neurogenesis changes in CA1 (Fig. 6G-I) or DG (not 

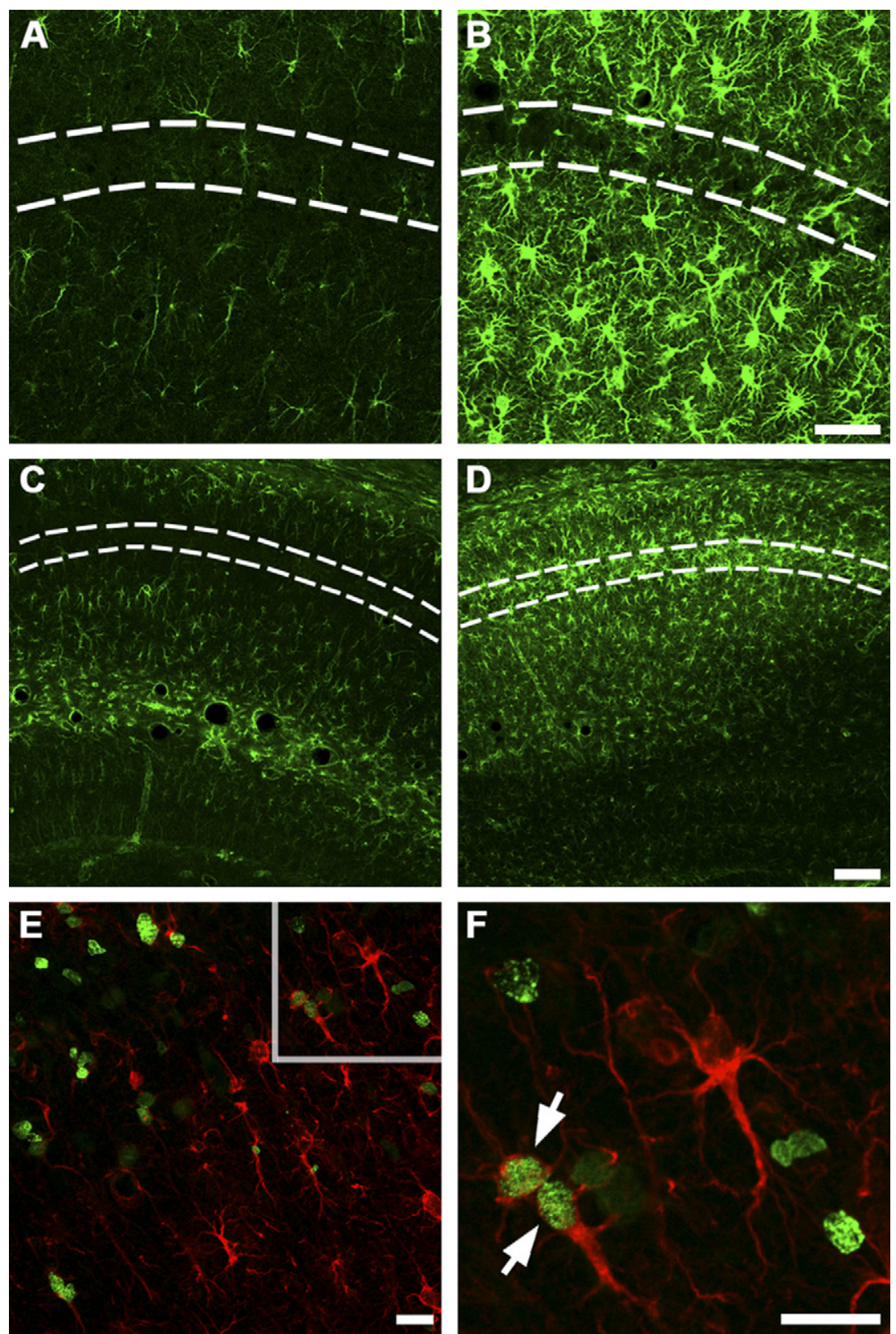

Fig. 4. Increased GFAP immunoreactivity in ischemic CA1 area. (A-D) Representative examples of single immunofluorescent labelling for GFAP (green), showing astrocytes in CA1 area (CA1 pyramidal cell layer is indicated by dashed lines). (A and C) Sham control animals at different magnification, (B) 5 days after ischemia, (D) 12 days after ischemia. Note the important GFAP immunoreactivity increase after ischemia. (E) confocal image of the double immunofluorescent staining for GFAP (red) showing astrocytes, and BrdU (green) that shows proliferative cells in CA1 area 5 days after ischemia. (F) Enlarged view of the region indicated by the box in (E). Note the double-positive cells indicated by arrows in (F). Also note that not all of the BrdU positive cells are astrocytes. Scale bars: A and B $=50 \mu \mathrm{m}, \mathrm{C}$ and $\mathrm{D}=100 \mu \mathrm{m}, \mathrm{E}$ and $\mathrm{F}=20 \mu \mathrm{m}$ (For interpretation of the references to color in this figure legend, the reader is referred to the web version of the article.).

shown) were observed. The contralateral hemispheres from the same brains (hemispheres with CA1 pyramidal neuronal death) showed however the proliferation and neurogenesis findings in CA1 and DG reported in this study.

Our semi-quantification of neurogenesis in the DG showed, in line with previous findings (Liu et al., 1998; Takagi et al., 1999; Yagita et al., 2001; Sharp et al., 2002; Kokaia and Lindvall, 2003), increased neurogenesis in the dentate gyrus of ischemic animals compared to sham controls (Fig. 7). The number of new neurons was not different between the two sham-control groups: a mean $( \pm$ S.E.M. $)$ value of $3.7 \pm 0.5$ new neurons/20037.19 $\mu \mathrm{m}^{2}$ of GCL was found. Ischemia increased this number to $8.4 \pm 1.2$ new neurons $/ 20037.19 \mu \mathrm{m}^{2}$ of GCL in the group of animals that received injections of BrdU during days $4,5,6$ and 7 post-ischemia. In the group of animals that received injections of BrdU during days 10, 11, 12 and 13 postischemia, neurogenesis increased to $12.3 \pm 1.7$ new neurons/ $20037.19 \mu \mathrm{m}^{2}$ of GCL. It should be kept in mind that 12 days after ischemia corresponded to the time point for maximal increase in proliferation in the DG, whereas 5 days after ischemia corresponded to the time point for the marked cellular proliferation observed in CA1. 

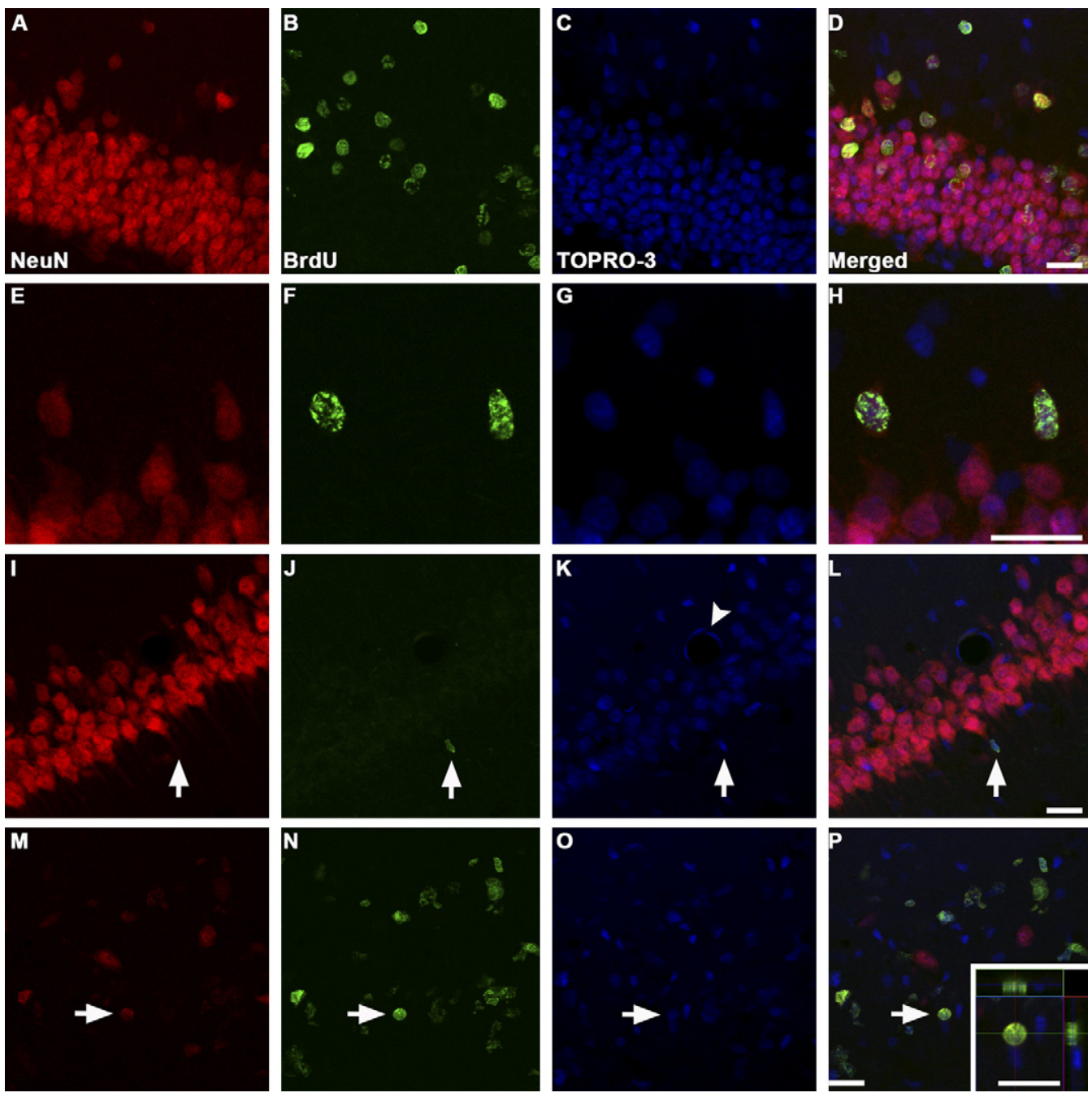

Fig. 5. Colocalization of NeuN-BrdU-TOPRO-3 in the DG and CA1. Representative confocal images of the immunofluorescent triple labelling for NeuN (red), BrdU (green) and TOPRO-3 (blue) showing the DG (A-H) and CA1 (I-P) 28 days after the first BrdU injection. (A-D) GCL of the DG 32 days after ischemia; (E-H) Enlargement by laser of an adjacent area; note the two triple positive cells in H, indicating new neurons. (I-L) CA1 pyramidal cell layer from sham-control animals; note that the $\mathrm{BrdU}(+)$ cell indicated by arrow in $(\mathrm{J}, \mathrm{K}$ and $\mathrm{L})$ do not express NeuN $(\mathrm{I})$, it may correspond to a new endothelial cell like those indicated by the arrowhead in (K). (M-P) CA1 pyramidal cell layer 32 days after ischemia; note the loss of neurons (NeuN staining) in ischemic animals compared to sham-controls (I-L) and the triple positive cell indicated by arrows (the right inset in $\mathrm{P}$ is a higher-magnification view and the 3D-analysis of this cell). We confirmed the validity of the triplepositive cells by scanning through three planes. All scale bars $=20 \mu \mathrm{m}$ (For interpretation of the references to color in this figure legend, the reader is referred to the web version of the article.).

\section{Discussion}

In the present study, we show that CA1 pyramidal cell layer death induces neurogenesis in dentate gyrus of gerbils and proliferative reaction in CA1 independently of the reduced cerebral blood flow.

One important issue with respect to the animal model used in this study is the presence of small vessels in the ventral surface of the midbrain that provide communication passages between the basilar and carotid circulations; this communication is sufficient to permit blood flowing into the carotid system despite bilateral CCA occlusion (Ginsberg and Busto, 1989). These connections may differ significantly from one side of the brain to the other side; which explains that seven animals did not show bilateral CA1 pyramidal neuronal death. Indian ink and monitoring of regional cerebral blood flow ( $\mathrm{rCBF}$ ) by laser
Doppler flowmetry have been used to mark the blood vessels in the gerbil brain and the presence of communicating arteries which correlate to an incomplete cessation of $\mathrm{rCBF}$ after CCA transient occlusion (Wang et al., 2002). In most gerbils, forebrain rCBF fell to near zero after CCA occlusion, and the percent decrease nearly reached $100 \%$. Because different degrees of arterial connections could be observed between the basilar and carotid circulations on one side, has been assigned grading score from 0 to $3: 0$, none; 1 , thin: 2 , medium; 3 , thick; indicate the degree of arterial connection; gerbils with score of 3 did not show delayed neuronal death in CA1 despite bilateral CCA occlusion. Nevertheless, these same animals (gerbils with score of 3) have a percent decline in forebrain rCBF of $75 \%$ (Wang et al., 2002) indicating that there is reduced cerebral blood flow (75\% less) but not CA1 neuronal death in the same forebrain. In our study, we found increased neurogenesis in the 

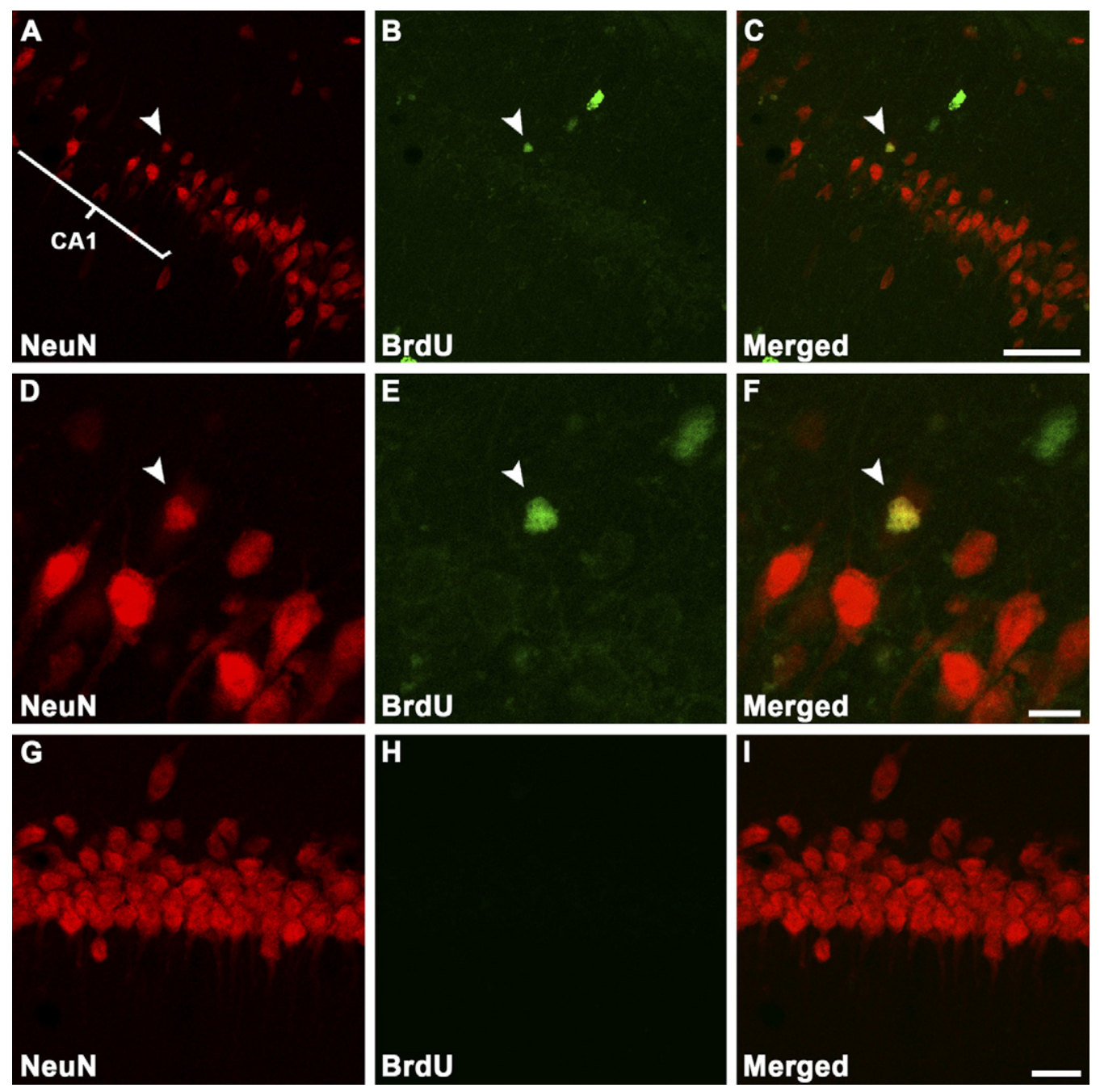

Fig. 6. CA1 32 days after ischemia. Representative confocal images from CA1 area of the hippocampus 32 days after ischemia, showing immunofluorescent staining for NeuN (red), BrdU (green) and merged. (A, B, C) Low magnification of CA1 pyramidal cell layer indicated by a line in (A); note the difference between the number of neurons in CA1 and in the rest of cell layers corresponding to CA2 and CA3 were there is not neuronal loss after ischemia. (D, E, F) Laser higher magnification of the same region. Note that the cell indicated by arrowheads in all panels is the same cell, demonstrating the colocalization of NeuN-BrdU in some cells that were found in the area of neuronal loss 32 days after ischemia. (G, H, I) CA1 pyramidal cell layer from one of the brain hemispheres subjected to ischemia but that not developed neuronal death. Note that there is no neuronal loss, proliferation or neurogenesis in this hippocampal region despite the bilateral CCA occlusion. Scale bars: A, B, $\mathrm{C}=50 \mu \mathrm{m} ; \mathrm{D}, \mathrm{E}, \mathrm{F}=10 \mu \mathrm{m} ; \mathrm{G}, \mathrm{H}, \mathrm{I}=20 \mu \mathrm{m}$ (For interpretation of the references to color in this figure legend, the reader is referred to the web version of the article.).

DG of ischemic animals that developed CA1 delayed neuronal death, which is widely known (Liu et al., 1998; Takagi et al., 1999; Yagita et al., 2001; Sharp et al., 2002; Kokaia and Lindvall, 2003), but in the 7 ischemic brain that did not show bilateral CA1 pyramidal neuronal death, the increased neurogenesis was observed only in the side of the brain that shows CA1 pyramidal neuronal death, demonstrated by loss of CA1 neurons in the same sections. Furthermore, all of the findings described in this paper in the ischemic groups (marked CA1 cell proliferation, increased proliferation in SGZ of the $\mathrm{DG}$, increase of reactive astrocytes in CA1 and DG, and increased neurogenesis in DG) were observed only on the side of the ischemic brains that developed CA1 death. We must keep in mind that even if these seven brains had the most score for degree of arterial connection (score 3) suggesting the presence of extensive communicating arteries, these brains will show a reduced forebrain rCBF of $75 \%$ after CCA occlusion. Then, we hypothesize that all of the findings described here, including the increased neurogenesis in the DG are a result of the CA1 pyramidal neuronal death and not of the reduced forebrain rCBF, so the ischemic pyramidal neuronal death in the CA1 subfield of the hippocampus could trigger neurogenesis independently of the reduced cerebral blood flow. This fact is important because it is indicating that the proliferation cell reactions and the neurogenesis described here are responses of the adult brain to the neuronal death, independently of the cause of the death and that it could occur in other neurodegenerative diseases that course with neuronal death. Liu et al. (1998) have reported that neurogenesis can occur in the DG of gerbils even without neural damage in CA1 after transient global ischemia; this apparent discrepancy may be explained by the different procedures and concepts used. The above authors used a model 

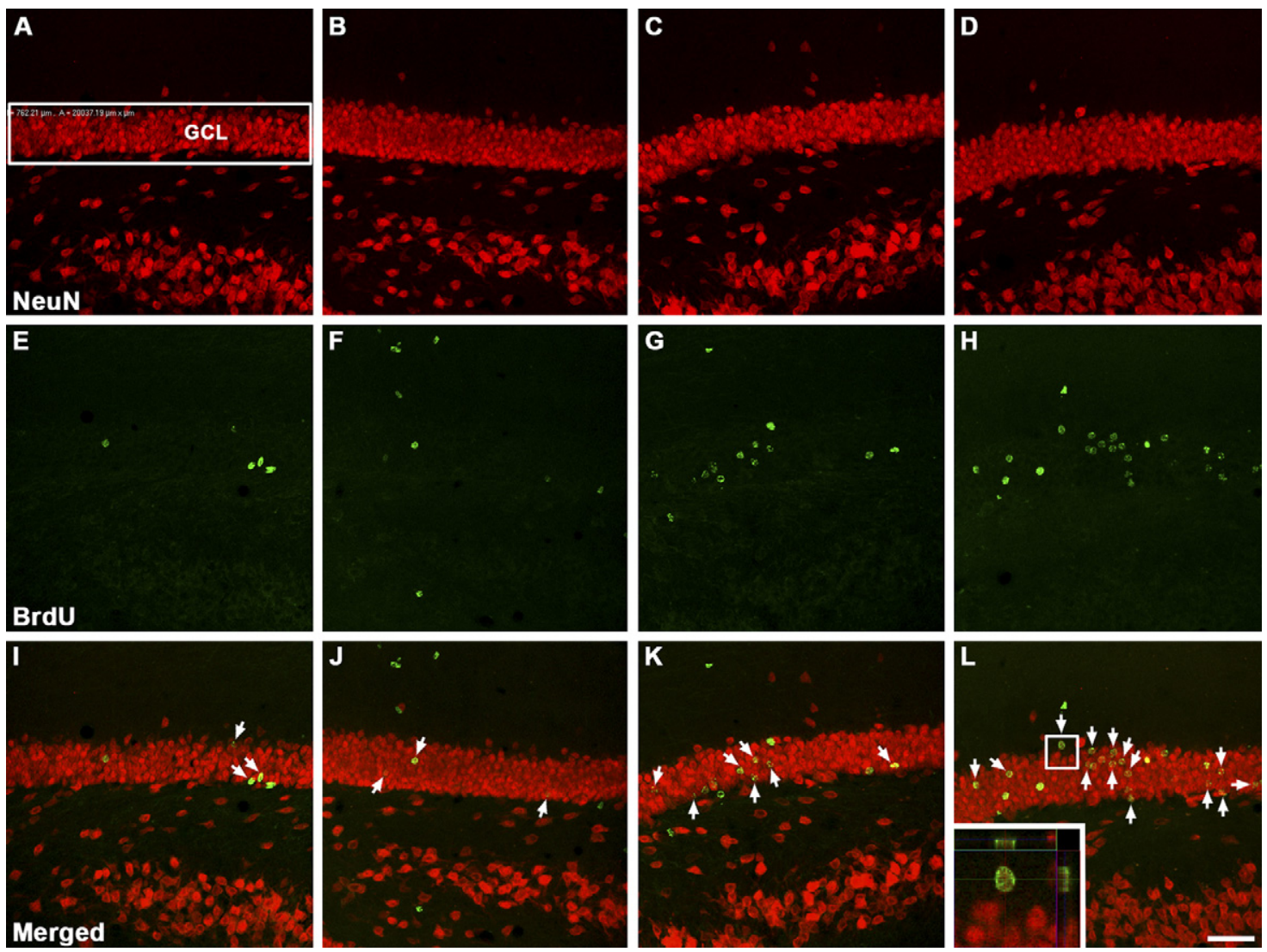

Fig. 7. Neurogenesis in the dentate gyrus. Representative confocal images of the immunofluorescent double labelling for NeuN (red), BrdU (green) and merged showing neurogenesis in GCL. The blue channel (TOPRO-3) is not shown here for better visualization. (A, E, I) Sham 32 days after surgery; (B, F, J) Sham 38 days after surgery; (C, G, K) 32 days after ischemia; (D, H, L) 38 days after ischemia. Animals sacrificed at 32 days received BrdU injections 4-7 days post surgery or ischemia. Animal sacrificed at 38 days received BrdU injections 10-13 days post surgery or ischemia. Newborn neurons, identified by morphology, anatomic localization and double positive labelling are indicated by arrows. Note the increase in the number of newborn neurons in case of ischemia (K and L) with respect to sham groups (I and J) and that it is maximal in (L). The left insert in (L) is a higher-magnification view and 3D-analysis of the cell in the box demonstrating the colocalization of NeuN-BrdU in this granular neuronal shaped cell. GCL: granule cell layer of the dentate gyrus indicated by the box in (A); the box also correspond to the sampling area of GCL used for counting $\left(20037.19 \mu \mathrm{m}^{2}\right.$ ). Scale bar $=50 \mu \mathrm{m}$ (For interpretation of the references to color in this figure legend, the reader is referred to the web version of the article.).

of ischemic tolerance: animals were preconditioned with $2 \mathrm{~min}$ of global ischemia and then subjected to $5 \mathrm{~min}$ of global ischemia $3 \mathrm{~d}$ later, BrdU was given $8 \mathrm{~d}$ after ischemia, and the animals were killed $1 \mathrm{~d}$ later. Then, they measured proliferation (number of BrdU positive cells $24 \mathrm{~h}$ after the injection of the mitotic marker), and not neurogenesis (new born neurons). Moreover, $\sim 50 \%$ of their animals appeared to have no CA1 neuronal loss.

Of particular importance when using BrdU administration to study cell proliferation in animals subjected to ischemia, is the possibility of incorporation of this thymidine analogue into cells committed to die. In these cells, a response to DNA damage involves nucleotide excision repair and, if this is undertaken in the presence of $\mathrm{BrdU}$, the cell can incorporate the nucleotide (Kuan et al., 2004). Hippocampal CA1 neurons are particularly sensitive and undergo selective, delayed degeneration in response to transient global ischemia (Kirino, 1982; Hara et al., 1993; Ferrer et al., 1997). Accordingly, we found a profound and selective loss of neurons, evaluated by Nissl staining (not shown) and NeuN labelling in this region $72 \mathrm{~h}$ after ischemia induction. However, at all time points considered in proliferation studies neither the pyramidal neurons of CA1 showed a colocalization of BrdU-NeuN nor the BrdU+ cells in CA1 5 days after ischemia had a normal pyramidal shape (Fig. 3J). With respect to neurogenesis studies, all the BrdU+ cells in the DG have appropriate shape and anatomic localization 28 days after first BrdU injection (Fig. 7). These observations suggest that the BrdU+ cells found in CA1 5 days after ischemia and in the DG 32 and 38 days after injury are new proliferating cells and do not correspond to preexisting neurons committed to die.

After ischemia in the gerbil, other studies have reported a marked increase in cell proliferation in SGZ of the DG, assessed by the number of cells labelled with BrdU in a $24 \mathrm{~h}$ period, which is maximal around 11 days after injury (Sharp et al., 2002). In line with these previous data, we found an enhanced cell proliferation in the SGZ, which was maximal at 12 days after ischemia, and, moreover a marked cell proliferation in gerbil CA1 subfield 5 days after ischemia. However, in the SVZ we did not find significant cell proliferation at the times considered after injury, although a few ectopic migrating cells could be detected; indicating that 
the proliferative reaction in CA1 did not correspond to migrating cells from SVZ as others have suggested (Gould et al., 1999; Schmidt and Reymann, 2002). Also, in our proliferation studies BrdU injection was performed $24 \mathrm{~h}$ before sacrifice, a too short time for cell migration from the DG or SVZ to CA1. In the DG, newly generated neurons migrate from SGZ to GCL to become granule neurons after approximately 28 days (Ming and Song, 2005).

The increased GFAP immunoreactivity found in the DG and CA1 from ischemic animals (Figs. 1N-P and 4, respectively) in agreement with other studies (Herrmann et al., 2000; Yasuda et al., 2004; Tatsumi et al., 2005) may correspond to activated astrocytes after ischemic insult. But although it is accepted that glial cells are activated after brain ischemia, also it is known that multipotent neural precursors have astrocytic morphology and express GFAP, i.e., that GFAP labelling may also correspond to adult neural stem cells (Sun et al., 2003; Alvarez-Buylla and Lim, 2004; Brazel and Rao, 2004; PicardRiera et al., 2004; Steiner et al., 2004; Ming and Song, 2005). In line with this, some of double BrdU-GFAP positive cells that were increased in CA1 5 days after ischemia may correspond to neural stem cells or neuronal resident progenitors. Only $32 \%$ of BrdU+ cells showed colocalization with GFAP 5 days after ischemia, indicating that there exists more than one cellular type in the proliferative reaction (Fig. 4). Another cell type that could be participating in this reaction is the microglia. Nevertheless, 32 days after ischemia we found triple NeuNBrdU-TOPRO-3 positive cells in CA1 (Figs. 5M-P and 6) although few. It is described that proliferating cells differentiate into neurons in the hippocampal CA1 region of gerbils after global cerebral ischemia (Schmidt and Reymann, 2002). Then, these cells could represent new neurons which might originate from progenitor cells in the parenchyma of this subfield. In situ activation of endogenous progenitors, located in both the CA1 periventricular region and parenchyma, after intraventricular infusion of FGF-2 and EGF leads to regeneration of pyramidal CA1 neurons after ischemic injury (Nakatomi et al., 2002). Several studies have also demonstrated the production of new neurons in regions other than SGZ or SVZ of the adult mammalian brain (Palmer et al., 1999; Gage, 2000; Magavi et al., 2000; Pencea et al., 2001; Yamamoto et al., 2001; Eisch, 2002; Tonchev et al., 2003; Emsley et al., 2005; Ming and Song, 2005). However to establish that these cells in CA1 are new neurons, that are integrated into the existing brain circuitry and contribute to ameliorating neurological deficits, more studies like electrophysiology and behavioural tests are needed.

On the basis of our findings in proliferation studies, we chose an adequate regime of BrdU administration allowing the study of the neurogenesis process in our experimental model (4-7 or 10-13 days post-ischemia). The increased neurogenesis found in GCL of the DG of ischemic animals was more marked in the group of animals that received injections of BrdU during days 10-13 post-ischemia (Fig. 7). Given that 12 days after injury correspond to the time point for maximal increase in SGZ proliferation (Figs. 1 and 2), it seems that this matches the increased neurogenesis in GCL. Colocalization of neurogenesis markers (NeuN-BrdU-TOPRO-3) and increase in the number of BrdU+ cells were detected in CA1 from ischemic animals that received BrdU injections 4-7 days post-ischemia and not in animals receiving BrdU 10-13 days post ischemia, which also matches the time of maximal proliferation ( 5 days) found in this region (Fig. 3).

In conclusion, this study support the notion that under appropriate conditions, neurogenesis may be mediated by endogenous adult neural progenitors resident in brain areas, such as CA1 or SGZ of the DG, and that this process is triggered in response to neuronal death. In this line, we hypothesize that this response would be independent of the cause of cell death. This opens up the possibility of attaining a treatment that enhances neurogenesis in situ against this or another class of neuronal death by manipulating these resident progenitors. The normal absence of constitutive neurogenesis does not reflect an intrinsic limitation for the potential of endogenous neural precursors, which may be activated by appropriate neurogenic intrinsic signals triggered by pathological conditions and, probably, also by exogenous manipulation. This may represent an interesting opportunity for regenerative therapies for stroke and other neurological diseases.

\section{Acknowledgment}

This work was supported by Ministerio de Educación y Ciencia, Spain (SAF-2003-07207-C02-02, SAF2005-05086) and UTE project FIMA.

\section{References}

Alvarez-Buylla, A., Lim, D.A., 2004. For the long run: maintaining germinal niches in the adult brain. Neuron 41, 683-686.

Brazel, C.Y., Rao, M.S., 2004. Aging and neuronal replacement. Ageing Res. Rev. 3, 465-483.

Eisch, A.J., 2002. Adult neurogenesis: implications for psychiatry. Prog. Brain Res. 138, 315-342.

Emsley, J.G., Mitchell, B.D., Kempermann, G., Macklis, J.D., 2005. Adult neurogenesis and repair of the adult CNS with neural progenitors, precursors, and stem cells. Prog. Neurobiol. 75, 321-341.

Ferrer, I., Ballabriga, J., Marti, E., Pozas, E., Planas, A.M., Blasi, J., 1997. BDNF and TrkB co-localize in CA1 neurons resistant to transient forebrain ischemia in the adult gerbil. J. Neuropathol. Exp. Neurol. 56, 790-797.

Gaese, B.H., Ostwald, J., 1995. Temporal coding of amplitude and frequency modulation in the rat auditory cortex. Eur. J. Neurosci. 7, 438-450.

Gaese, B.H., Ostwald, J., 2001. Anesthesia changes frequency tuning of neurons in the rat primary auditory cortex. J. Neurophysiol. 86, 1062-1066.

Gage, F.H., 2000. Mammalian neural stem cells. Science 287, 1433-1438.

Ginsberg, M.D., Busto, R., 1989. Rodent models of cerebral ischemia. Stroke 20, 1627-1642.

Gould, E., Tanapat, P., Hastings, N.B., Shors, T.J., 1999. Neurogenesis in adulthood: a possible role in learning. Trends Cogn. Sci. 3, 186-192.

Gratzner, H.G., 1982. Monoclonal antibody to 5-bromo- and 5-iododeoxyuridine: a new reagent for detection of DNA replication. Science 218, 474475.

Hara, H., Sukamoto, T., Kogure, K., 1993. Mechanism and pathogenesis of ischemia-induced neuronal damage. Prog. Neurobiol. 40, 645-670.

Herrmann, M., Vos, P., Wunderlich, M.T., de Bruijn, C.H., Lamers, K.J., 2000. Release of glial tissue-specific proteins after acute stroke: A comparative analysis of serum concentrations of protein S-100B and glial fibrillary acidic protein. Stroke 31, 2670-2677.

Horiguchi, T., Shima, H., Suga, S., Ogino, M., Shimizu, K., Toya, S., Nagao, M., Kawase, T., 2002. Transient forebrain ischemia induces expression of 
serine/threonine protein phosphatase $1 \mathrm{mRNA}$ in the vulnerable regions of gerbil brain. Neurosci. Lett. 325, 115-118.

Kirino, T., 1982. Delayed neuronal death in the gerbil hippocampus following ischemia. Brain Res. 239, 57-69.

Kokaia, Z., Lindvall, O., 2003. Neurogenesis after ischaemic brain insults. Curr. Opin. Neurobiol. 13, 127-132.

Kuan, C.Y., Schloemer, A.J., Lu, A., Burns, K.A., Weng, W.L., Williams, M.T., Strauss, K.I., Vorhees, C.V., Flavell, R.A., Davis, R.J., Sharp, F.R., Rakic, P., 2004. Hypoxia-ischemia induces DNA synthesis without cell proliferation in dying neurons in adult rodent brain. J. Neurosci. 24, 10763-10772.

Kuhn, H.G., Dickinson-Anson, H., Gage, F.H., 1996. Neurogenesis in the dentate gyrus of the adult rat: age-related decrease of neuronal progenitor proliferation. J. Neurosci. 16, 2027-2033.

Liu, J., Solway, K., Messing, R.O., Sharp, F.R., 1998. Increased neurogenesis in the dentate gyrus after transient global ischemia in gerbils. J. Neurosci. 18, 7768-7778.

Magavi, S.S., Leavitt, B.R., Macklis, J.D., 2000. Induction of neurogenesis in the neocortex of adult mice. Nature 405, 951-955.

Martin, R.M., Leonhardt, H., Cardoso, M.C., 2005. DNA labeling in living cells. Cytometry A 67, 45-52.

Ming, G.L., Song, H., 2005. Adult neurogenesis in the mammalian central nervous system. Annu. Rev. Neurosci. 28, 223-250.

Nakatomi, H., Kuriu, T., Okabe, S., Yamamoto, S., Hatano, O., Kawahara, N., Tamura, A., Kirino, T., Nakafuku, M., 2002. Regeneration of hippocampal pyramidal neurons after ischemic brain injury by recruitment of endogenous neural progenitors. Cell 110, 429-441.

Palmer, T.D., Markakis, E.A., Willhoite, A.R., Safar, F., Gage, F.H., 1999. Fibroblast growth factor-2 activates a latent neurogenic program in neural stem cells from diverse regions of the adult CNS. J. Neurosci. 19, 8487-8497.

Pencea, V., Bingaman, K.D., Wiegand, S.J., Luskin, M.B., 2001. Infusion of brain-derived neurotrophic factor into the lateral ventricle of the adult rat leads to new neurons in the parenchyma of the striatum, septum, thalamus, and hypothalamus. J. Neurosci. 21, 6706-6717.

Picard-Riera, N., Nait-Oumesmar, B., Baron-Van Evercooren, A., 2004. Endogenous adult neural stem cells: limits and potential to repair the injured central nervous system. J. Neurosci. Res. 76, 223-231.

Schmidt-Kastner, R., Freund, T.F., 1991. Selective vulnerability of the hippocampus in brain ischemia. Neuroscience 40, 599-636.
Schmidt, W., Reymann, K.G., 2002. Proliferating cells differentiate into neurons in the hippocampal CA1 region of gerbils after global cerebral ischemia. Neurosci. Lett. 334, 153-156.

Sharp, F.R., Liu, J., Bernabeu, R., 2002. Neurogenesis following brain ischemia. Brain Res. Dev. Brain Res. 134, 23-30.

Steiner, B., Kronenberg, G., Jessberger, S., Brandt, M.D., Reuter, K., Kempermann, G., 2004. Differential regulation of gliogenesis in the context of adult hippocampal neurogenesis in mice. Glia 46, 41-52.

Sun, Y.E., Martinowich, K., Ge, W., 2003. Making and repairing the mammalian brain-signaling toward neurogenesis and gliogenesis. Semin. Cell Dev. Biol. 14, 161-168.

Suzuki, T., Fujikura, K., Higashiyama, T., Takata, K., 1997. DNA staining for fluorescence and laser confocal microscopy. J. Histochem. Cytochem. 45, 49-53.

Takagi, Y., Nozaki, K., Takahashi, J., Yodoi, J., Ishikawa, M., Hashimoto, N., 1999. Proliferation of neuronal precursor cells in the dentate gyrus is accelerated after transient forebrain ischemia in mice. Brain Res. 831, 283-287.

Tatsumi, K., Haga, S., Matsuyoshi, H., Inoue, M., Manabe, T., Makinodan, M., Wanaka, A., 2005. Characterization of cells with proliferative activity after a brain injury. Neurochem. Int. 46, 381-389.

Tonchev, A.B., Yamashima, T., Zhao, L., Okano, H.J., Okano, H., 2003. Proliferation of neural and neuronal progenitors after global brain ischemia in young adult macaque monkeys. Mol. Cell Neurosci. 23, 292-301.

Wang, Q., Xu, J., Rottinghaus, G.E., Simonyi, A., Lubahn, D., Sun, G.Y., Sun, A.Y., 2002. Resveratrol protects against global cerebral ischemic injury in gerbils. Brain Res. 958, 439-447.

Yagita, Y., Kitagawa, K., Ohtsuki, T., Takasawa, K., Miyata, T., Okano, H., Hori, M., Matsumoto, M., 2001. Neurogenesis by progenitor cells in the ischemic adult rat hippocampus. Stroke 32, 1890-1896.

Yamamoto, S., Yamamoto, N., Kitamura, T., Nakamura, K., Nakafuku, M., 2001. Proliferation of parenchymal neural progenitors in response to injury in the adult rat spinal cord. Exp. Neurol. 172, 115-127.

Yasuda, Y., Tateishi, N., Shimoda, T., Satoh, S., Ogitani, E., Fujita, S., 2004. Relationship between S100beta and GFAP expression in astrocytes during infarction and glial scar formation after mild transient ischemia. Brain Res. $1021,20-31$. 